\title{
COMPACTNESS PROPERTIES OF A LOCALLY COMPACT GROUP AND ANALYTIC SEMIGROUPS IN THE GROUP ALGEBRA*
}

\author{
JosÉ E. GALÉ
}

\section{0 . Introduction}

Let $G$ be a locally compact group with left Haar measure $\mu$, and let $L^{1}(G)$ be the convolution Banach algebra of integrable functions on $G$ with respect to $\mu$. In this paper we are concerned with the investigation of the structure of $G$ in terms of analytic semigroups in $L^{1}(G)$. In this context, a well known result is the theorem of $A$. Sinclair which says that $G$ is metrizable if and only

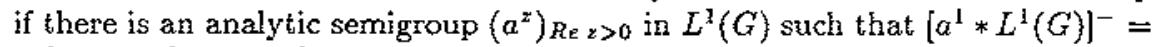
$\left[L^{1}(G) * a^{1}\right]^{-}=L^{1}(G)([26$, p.41]). Other results relating the behavior of analytic semigroups on half-discs to certain linear properties of $L^{\ddagger}(G)$ have been obtained in [6]. The following problem was raised by J. Estcrle in $[3$, p.460].

Question E. If $L^{1}(G)$ has a non-zero analytic semigroup $\left(a^{z}\right)_{\text {He } z>0}$ which is bounded on the line $\{\operatorname{Re} z=1\}$, does it follow that $G$ is compact? What about the converse?

Also, A. Sinclair asked in [26] for the relationships between the rate of growth on vertical lines of analytic semigroups in $L^{1}(G)$, and compactness properties of $G$. Recall that $G$ is said to be of polynomial growth if for every compact neighborhood $K$ of $G$ there is a nonnegative integer $m$ such that

$$
\mu\left(K^{n}\right)=O\left(n^{m}\right) \text { as } n \rightarrow \infty
$$

([23, p.280]). If the minimal $m$ for which $(*)$ is satisfied is the same for all compacts $K$ then this number is called the degree of growth in $G$. Such a number exists if, for example, $G$ is connected or $G$ is compact $(m=0$ in this

*This research was started, and a part of the results were obtained, during the attendence of the author at "Semester on Automatic Continuity and Banach Algebras" in the University of Leeds, Iune 1987, supported by the U. K. Science and Engineering Research Council. The research has also been partially supported by the Spanish DGICYT, Proyecto PS87-0059, and the Caja de Ahorros de la Inmaculada, Zaragoza, Ayuda CB 11/88 
last case). Then, does $G$ have polynomial growth if and only if there is an analytic semigroup $\left(a^{z}\right)_{R e z>0}$ in $L^{1}(G)$ and a nonnegative integer $N$ such that $\left\|a^{1+i y}\right\|=O\left(|y|^{N}\right)$ as $|y| \rightarrow \infty, y \in \mathbb{R}$ ? (Sec $[\mathbf{2 6}, \mathrm{p} .81]$ ). Recently, T. Pytlik has shown that if an element $b$ of a Banach algebra $A$ is such that $\left\|e^{i t b}\right\|=O\left(|t|^{N}\right)$, as $|t| \rightarrow \infty(t \in \mathbb{R})$ for some nonnegative integer $N$, then there exists an analytic semigroup $\left(a^{z}\right)_{R e z>0}$ in $A$ such that $\left\|a^{1+i y}\right\|=O\left(|y|^{N+1}\right)$ as $|y| \rightarrow \infty(y \in \mathbb{R})$ ([25]). J. Dixmier had proved in $[8$, p.17] that, for a group $G$ of polynomial growth, such an element $b$ exists in $L^{1}(G)$ and, furthermore, the number $N$ associated to $b$ can be chosen as $N=m+1$, if $m$ satisfies condition (*). Thus Sinclair's question has an affirmative answer in one way, but as far as we know nothing else is known about the converse. Notice that the first part of Question $E$ can be viewed as a particular case of this converse.

Here, we present a first stage in the study of Question $E$. We prove that, if $G$ is a central group, then $L^{1}(G)$ has a non-zero analytic scmigroup $\left(a^{z}\right)_{R e} z>0$ such that $\left\{a^{1+i y}: y \in \mathbb{R}\right\}$ is relatively weakly compact if and only if $G$ contains a compact, open subgroup. If, furthermore, $G$ is also connected then $G$ must be compact. We obtain this theorem as a consequence of the following one, which we prove in a more abstract setting : if $\left(a^{z}\right)_{\text {Re. } z>0}$ is an analytic semigroup in a Banach algebra $A$ such that $\left\{a^{1+i y}: y \in \mathbb{R}\right\}$ is relatively weakly compact, then the spectrum $\sigma\left(a^{\mathrm{I}}\right)$ is at most countable. The proof of this last result which we give here is based upon elementary properties of the weakly almost periodic functions on the real line (see $[9],[10]$ ). Other essential ingredients in our general argument are the structural properties of central groups ([17\},\{18]).

We also show that for every locally compact group $G$ containing a compact and open subgroup, $L^{1}(G)$ has an analytic semigroup which is norm compact on $\{R e z=1\}$. Then, if $G$ is central (in this case $G$ is of polynomial growth) its structure allows us to improve the number $N$ appearing in Sinclair's question, with respect to the one we would get from the theorems given by Dixmier and Pytlik in [8] and [25], respectively.

The paper is divided into three sections. In the first one, we collect definitions and basic properties of weakly almost periodic functions, analytic semigroups in Banach algebras, and central groups, that we shall need in the two other sections. Section 2 is devoted to prove the result mentioned above for analytic semigroups in Banach algebras. In section 3 we discuss Question $E$, and we give there the precedings results about group algebras.

Acknowledgements. We wish to thank B. Aupetit, O. Blasco, J. C. Candeal, B. Cuartero, J. Esterle, N. Grønbæk, T. Ransford for helpful conversations or comments about subjects of this paper, and A. Hulanicki and T. Pytlik for reprints.

\section{Preliminaries}

Let $\mathbb{R}$ be the real line, and denote by $C_{b}(\mathbb{R})$ the Banach algebra of bounded and continuous functions on $\mathbb{R}$. If $f \in C_{b}(\mathbb{R})$ and $t \in \mathbb{R}$ we define $f_{t} \in C_{b}(\mathbb{R})$ 
by $f_{t}(s)=f(s+t)(s \in \mathbb{R})$. A function $f \in C_{b}(\mathbb{R})$ is said to be weakly almost periodic if $\left\{f_{t}: t \in \mathbb{R}\right\}$ is relatively compact in the weak topology of $C_{b}(\mathbb{R})$. This notion, which was introduced by W. Eberlein in [9], is a generalization of the well known almost periodic functions of $\mathrm{H}$. Bohr ([4]). Denote by $W(\mathbf{R})$ the class of weakly almost periodic functions on $\mathbb{R}$. Every continuous function on which is null at infinity, and every positive definite function on $\mathbb{R}$ belong to $W(\mathbb{R})$. The class $W(\mathbb{R})$ enjoys interesting properties. We shall only need the following mean ergodic theorem ([9, Theorem 15.2])

Theorem 1.1. If $f \in W(\mathbb{R})$ and $\lambda \in \mathbb{R}$, the mean value

$$
M_{\lambda}(f)=\lim _{r \rightarrow \infty}(1 / r) \int_{0}^{r} f(t) e^{-i \lambda t} d t
$$

exists. Moreover, $\left\{\lambda \in \mathbb{R}: M_{\lambda}(f) \neq 0\right\}$ is at most countable.

Let $H$ be the open right-hand half-plane of $\mathbb{C}$, and let $A$ be a Banach algebra. An analytic semigroup in $A$ is an analytic function $z \rightarrow a^{z}, H \rightarrow A$ such that $a^{z+w}=a^{z} a^{w}(z, w \in H)$. We identify an analytic semigroup with its range, denoted by $\left(a^{z}\right)_{R e z>0}$. Throughout, whenever we consider analytic semigroups, we shall write $a$ instead of $a^{3}$. We are interested in a non-zero analytic semigroup $\left(a^{z}\right)_{R e z>0}$ with $\sup _{y \in \mathbb{R}}\left\|a^{3+i y}\right\|<\infty$. In this case, it is well known that if $A$ is commutative, and $\Phi_{A}$ is the character space of $A$, then for each $\varphi \in \Phi_{A}$ there is $\alpha \in \mathbb{R}$ such that $\varphi\left(a^{z}\right)=e^{\alpha z}(z \in H)$ (sec [26, p.85]). Also, the Banach algebra generated by the semigroup equals the Banach algebra polynomially generated by $a$ ([14, p.379]).

Let $G$ be a locally compact group, and let $Z(G)$ be its centre, that is, $Z(G)=$ $\{t \in G: t s=s t$ for all $s \in G\}$. The group $G$ is said to be central if the quotient group $G / Z(G)$ is compact. Clearly, all compact groups and all locally compact. abelian groups are central. "In fact, many of the features common to these two classes appear in their natural setting only when viewed as being characteristic of central groups. In addition there are strong indications that the class of central groups marks the utmost degree of generality in which all these features are still present" $([18$, p.361]). We shall use the following results in section 3

Theorem 1.2. Let $G$ be a central group. Then there exists $n \geq 0$ such that $G \cong \mathbb{R}^{n} \times G_{0}$ where $G_{0}$ is a locally compact group containing a compact, open, normal, subgroup $K$ such that $G_{0} / K$ is abelian.

A proof of this is in [17, p.331].

The class of all connected, central groups admits several interesting, and equivalent, descriptions ([21, p.14,15]). We shall only need the next one

Theorem 1.3. If $G$ is a connected central group then $G \cong \mathbb{R}^{n} \times K$, where $n \geq 0$ and $K$ is a compact (connected) group. 
The corresponding resuits on the structure of locally compact abelian groups can be seen in [20 I].

We finish this section with a simple observation

Lemma 1.4. If $G$ is central, then $G$ is of polynomial growth.

Proof: The centre $Z(G)$ is of polynomial growth because it is commutative ([12, Theorem 7.8]). Besides that, $G / Z(G)$ is compact by definition. Then the result follows from $[22$, p. 167,168$]$.

\section{Analytic semigroups which are relatively weakly compact on vertical lines}

The next proposition is a crucial step in our reasoning

Proposition 2.1. Let $A$ be a Banach algebra, and let $A^{*}$ be its dual Banach space. Suppose that there is an analytic semigroup $\left(a^{*}\right)_{R e}>0$ in $A$ such that $\left\{a^{1+i y}: y \in \mathbb{R}\right\}$ is relatively weakly compact. Then

(i) For every $\varphi \in A^{*}$, the function $y \rightarrow \varphi\left(a^{2+i y}\right), \mathbb{R} \rightarrow \mathbb{C}$ is weakly almost periodic.

(ii) The weak limit

$$
\lim _{r \rightarrow \infty}(1 / r) \int_{0}^{r} a^{2+i y} e^{-i \lambda y} d y
$$

exists in $A$ for each $\lambda \in \mathbb{R}$, and so it defines an element $b_{\lambda}$ of $A$.

(iii) The set $\left\{\lambda \in \mathbf{R}: b_{\lambda} \neq 0\right\}$ is at most countable.

Proof: (i) Put $f(y)=\varphi\left(a^{2+i y}\right)(y \in \mathbb{R})$ and $\Psi(b)(y)=\varphi\left(a^{1+i y} b\right)(b \in A, y \in$ R). Then $\Psi$ is a continuous linear function from $A$ to $C_{b}(\mathbb{R})$, which is also continuous with respect to the corresponding weak topologies, and we have that $f_{v}=\Psi\left(a^{1+i t}\right)(v \in \mathbf{R})$. It follows that $f \in W(\mathbf{R})$.

(ii) For each $\lambda \in \mathbb{R}$, the function $y \rightarrow a^{2+i y} e^{-i \lambda y}, \mathbf{R} \rightarrow A$ is continuous and then it is Bochner integrable over each interval $[0, r](r \in \mathbf{R})$, and its range $A_{0}$ is separable. Put

$$
b_{r, \lambda}=(1 / r) \int_{0}^{r} a^{2+i y} e^{-i \lambda y} d y(\lambda, r \in \mathbb{R})
$$

By (i), there exists $b_{\lambda} \in A^{* *}$ such that $\left\langle b_{\lambda}, \varphi\right\rangle=\lim _{r \rightarrow \infty} \varphi\left(b_{r, \lambda}\right)$. But, for each $r \in \mathbf{R}, b_{\lambda}$ belongs to the (weakly) closed convex hull $C_{\lambda}$ of the set $\left\{a^{2+i y} e^{-i \lambda y}\right.$ : $y \in \mathbf{R}\} \subset A_{0}$. Then $C_{\lambda}$ is a weakly compact subset of $A_{0}$, by the Krein's Theorem ([11, p.553]). Since $b_{\lambda}$ belongs to $C_{\lambda}$, we obtain that $b_{\lambda} \in A_{0}$.

(iii) Since $A_{0}$ is separable we can choose a sequence $\left(\varphi_{n}\right)_{n \geq 1}$ in $A_{0}^{*}$ such that $\|b\|=\sup _{n \geq 1}\left|\varphi_{n}(b)\right|$ for every $b \in A_{0}$. Let $X_{n}$ be a countable subset of 
$\mathbb{R}$ such that $\varphi_{n}\left(b_{\lambda}\right)=0$ if $\lambda \notin X_{n}$. Set $X=\bigcup_{n \geq 1} X_{n}$. Then, if $\lambda \notin X$, $\left\|b_{\lambda}\right\|=\sup _{n \geq 1}\left|\varphi_{n}\left(b_{\lambda}\right)\right|=0$.

The arguments which we have considered to prove parts (i), (ii), and (iii) of the above proposition have been taken from $[16, p .82,83],[16, p .84]$, and $[1$, p.43], respectively. They have been written in its present form in order to give a more elementary proof of such properties, in this context.

The following theorem is the key point of this paper.

Theorem 2.2. Let $A$ be a Banach algebra which contains an analytic semigroup $\left(a^{2}\right)_{\text {Re } z>0}$ such that $\left\{a^{1+i y}: y \in \mathbb{R}\right\}$ is relatively weakly compact. Then the spectrum $\sigma(\alpha)$ of $a$ is at most countable.

Proof: We can assume that $A$ is generated as Banach algebra by the semigroup $\left(a^{z}\right)_{R e}>0$. By Proposition 2.1, $\left\{\lambda \in \mathbb{R}: b_{\lambda} \neq 0\right\}$ is at most countable, where

$$
b_{\lambda}=\text { weak }-\lim _{r \rightarrow \infty}(1 / r) \int_{0}^{r} \alpha^{2+i y} e^{-i \lambda y} d y(\lambda \in \mathbb{R})
$$

If $\varphi$ is a character of $A$ we have that $\varphi\left(a^{z}\right)=e^{\alpha z}(z \in H)$, for some $\alpha \in \mathbf{R}$. Then,

$$
\begin{gathered}
\varphi\left(b_{\alpha}\right)=\lim _{r \rightarrow \infty}(1 / r) \int_{0}^{r} \varphi\left(a^{2+i y}\right) e^{-i \alpha y} d y \\
=\lim _{r \rightarrow \infty}(1 / r) \int_{0}^{r} e^{2 a} e^{i \alpha y} e^{-i \alpha y} d y=e^{2 a} \neq 0 .
\end{gathered}
$$

Thus, $\sigma(a)$ is at most countable.

A more formal proof of this fact is also available by using some theorems on compact topological semigroups (see [15] for this topic). Such a proof relies heavily on ideas coming from the basic theory of regular quasimultipliers of Banach algebras, which can be seen in [13]. The proof, and more information about commutative Banach algebras generated by semigroups as in Theorem 2.2 , are given in $[27]$.

Corollary 2.3. Let $A$ be a non-unital, commutative, semisimple, Banach algebra. Suppose that its character space $\Phi_{A}$ is connected in the Gelfand topology. If $\left(\alpha^{z}\right)_{R e z}>0$ is an analytic semigroup in $A$ such that $\left\{a^{\mathrm{I}+i y}: y \in \mathbb{R}\right\}$ is relatively weakly compact then $a=0$.

Proof: By the hypothesis on $A, \Phi_{A}$ is non compact ([24, p.154]). Also, the function $\varphi \rightarrow \varphi(a), \Phi_{A} \rightarrow \mathbb{C}$ is continuous and null at infinity, whence $0 \in\left\{\varphi \in \Phi_{A}\right\}^{-}$. Moreover, for each $\varphi \in \Phi_{A}$ there is $\alpha \in \mathbf{R}$ with $\varphi(a)=e^{\alpha}$. Thus the spectrum $\sigma(a)$ of $a$ is a connected subset of $\mathbf{R}$. But $\sigma(a)$ is also countable (Theorem 2.2), and therefore $\sigma(a)=0$. 
Analogous arguments to those of the proof of this corollary will be used to prove Theorem 3.3 below.

Remark. The condition on the semigroup in the above results camot be rcplaced by the weaker assumption that $\sup _{y \in \mathbb{*}}\left\|a^{\mathrm{I}+i y}\right\|<\infty$ : let $A=C_{0}([0,1]\}$, the Banach algebra of continuous functions on $[0,1]$ which are null at $t=0$. Set $a^{z}(t)=e^{z \log t}$, where $\log t$ is the branch of the $\operatorname{logarithm}$ which is real-valued on the positive real numbers. Then $\left(a^{z}\right)_{R e z}>0$ is an analytic semigroup in $A$ such that $\sup _{y \in \mathbb{R}}\left\|a^{1+i y}\right\|<\infty$, but $\sigma(a)=[0,1]$.

\section{Group algebras}

We begin by giving a result concerning the second part of Question E. Let $G$ be a locally compact group, and let $G_{0}$ be an open subgroup of $G$. It is straightforward to verify that the mapping $\psi: f \rightarrow \psi(f), L^{1}\left(G_{0}\right) \rightarrow L^{1}(G)$ given by $\psi(f)(t)=f(t)\left(t \in G_{0}\right)$, and $\psi(f)(t)=0\left(t \in G-G_{0}\right)$ is a continuous algebra homomorphism. On the other hand, if $G$ is a compact group then there are sequences $\left(\varphi_{n}\right)_{n \geq 1}$ of functions on $G$ such that $\left(\varphi_{n}\right)_{n \geq 1} \subset L^{1}(G),\left\|\varphi_{n}\right\| \leq 1$, $\varphi_{n} * \varphi_{m}=\delta_{n m} \varphi_{n}(n, m \geq 1)([20$ II, p.14])

Proposition 3.1. Let $G$ be a locally compact group containing a compact, open subgroup. Then $L^{1}(G)$ has a non-zero analytic semigroup $\left(a^{z}\right)_{R e} z>0$ such that $\left\{a^{1+i y}: y \in \mathbb{R}\right\}$ is norm compact.

Proof: Let us assume that $G$ is compact and take a sequence $\left(\varphi_{n}\right)_{n \geq 1}$ as before the proposition. Recall that $H$ is the open right half plane. Put $a^{z}(t)=$ $\sum_{n \geq 1} e^{-n x} \varphi_{n}(t)(t \in G, z \in H)$. Take $\alpha>0$ and $z \in H$ such that $R e z \geq \alpha$. Then $\left\|e^{-n z} \varphi_{n}\right\| \leq e^{-n R e} z \leq e^{-a n}$. Hence the function $z \rightarrow a^{2}, H \rightarrow L^{1}(G)$ is analytic, and it is also clear that $a^{z} * a^{w}=a^{x+w}(z, w \in H)$. Furthermore, since $a^{1+i y}=a^{1+i(y+2 \pi)}(y \in \mathbf{R})$, the continuity of the mapping $y \rightarrow a^{1+i_{y}}, \mathbb{R} \rightarrow$ $L^{1}(G)$ inplies the compactness of $\left\{a^{1+i y}: y \in[0,2 \pi]\right\}$

Consider now the general case and let $G_{0}$ be a compact, open, subgroup of $G$. Let $\left(a^{z}\right)_{R e z>0}$ be the sernigroup in $L^{\mathrm{I}}\left(G_{0}\right)$ given before. Put $b^{z}=\psi\left(a^{z}\right)\langle z \in$ $H$ ), where $\psi: L^{1}\left(G_{0}\right) \rightarrow L^{1}(G)$ is as before. Clearly, $\left(b^{z}\right)_{R e z>0}$ satisfies the required conditions.

As Proposition 3.1 shows, there exist non compact locally compact groups $G$ such that $L^{1}(G)$ has non-zero analytic sernigroups which are not only bounded, but compact, on $\{\operatorname{Re} z=1\}$.

We can give a partial converse to the above proposition. But, before doing this, let us say at this point some words about the question suggested by $A$. Sinclair (see Introduction). As already said, if $G$ is a group of polynomial growth, and if $m$ is a nomegative integer such that

$$
\mu\left(K^{n}\right)=O\left(n^{m}\right) \text { as } n \rightarrow \infty
$$


for some compact neighborkood $K$ in $G$, then the results given by Dixmier and $P y t l i k$ in $[8]$ and [25] imply that there is a non-zero analytic semigroup $\left(a^{z}\right)_{\operatorname{Re} z>0}$ in $L^{1}(G)$ such that $\left\|a^{1+i y}\right\|=O\left(|y|^{N}\right)$ as $|y| \rightarrow \infty(y \in \mathbb{R})$, with $N=m+2$. If $G$ is central, we can improve substantially the relationship between $m$ and the growth of $\left\|a^{1+i y}\right\|$ at the infinity, by using the structure of $G$ and the same ideas as above.

Proposition 3.2. Let $G$ be a central group and let $m$ be the minimal nonnegative integer for which the condition (*) holds. Then there is a non-zero analytic semigroup $\left(a^{x}\right)_{R e} z>0$ in $L^{1}(G)$ such that $\left\|a^{1+i y}\right\|=O\left((\log |y|)^{m}\right)$ as $|y| \rightarrow \infty(y \in \mathbf{R})$, if $m \geq 1$.

Proof: By Theorem 1.2, $G \cong \mathbb{R}^{n} \times G_{0}$ where $n \geq 0$ and $G_{0}$ contains a compact, open subgroup. The degree of growth of $\mathbf{R}^{n}$ is $n([12, \mathrm{p} .181])$, and so $n=m$. Take in $L^{1}(\mathbb{R})$ the Poisson semigroup $\left(P^{x}\right)_{\operatorname{Re} z>0}$, for which $\left\|P^{1+i y}\right\|=$ $O(\log |y|)$ as $|y| \rightarrow \infty\left(\left[26\right.\right.$, p. 25,29]). Choose an analytic semigroup $\left(c^{z}\right)_{R e} z>0$ in $L^{1}\left(G_{0}\right)$ as the one given in Proposition 3.1. Then, if $a^{z}=P^{z} \otimes \ldots \otimes P^{z} \otimes c^{z}(z \in$ $H)$, the semigroup $\left(a^{z}\right)_{R e z>0}$ is in the tensor product $L^{1}\left(\mathbb{R}^{m}\right) \otimes L^{1}\left(G_{0}\right) \subset$ $L^{1}(G)$, and it satisfies the properties of the statement.

Next, we are going to show our main results concerning Question $E$. Let $A$ be a Banach algebra. If $X$ is a Banach space we denote by $\mathcal{L}(X)$ the space of continuous endomorphisms on $X$ endowed with the uniform norm. Recall that a representation $\varphi$ of $A$ on $X$ is a continuous algebra homomorphism $\varphi: a \rightarrow \varphi(a), A \rightarrow \mathcal{L}(X)$. A closed subspace $X_{1}$ of $X$ is said to be invariant under $\varphi$ if $\varphi(a)\left(X_{1}\right) \subset X_{1}$ for all $a \in A$. If $(0)$ and $X$ are the only closed subspaces of $X$ that are invariant under $\varphi$, and $\varphi$ is non null, then $\varphi$ is said to be irreducible. If $X$ is finite-dimensional, then $\varphi$ is called finite-dimensional $([5])$. We denote by $\mathcal{F}$ the set of finite-dimensional irreducible representations of $A$. We say that $A$ is $\mathcal{F}$-semisimple if $\varphi(b)=0$ for every $\varphi \in \mathcal{F}$ and some $b \in A$ implies that $b=0$.

Let us consider the convolution Banach algebra $L^{1}\left(\mathbb{R}^{n} ; A\right)$ of $A$-valued Bochner integrable functions on $\mathbb{R}^{n}$.

Theorem 3.3. Let $n \geq 1$, and let $A$ be a $\mathcal{F}$-semisimple Banach algebra. If $\left(a^{z}\right)_{R e} z>0$ is an analytic semigroup in $L^{1}\left(\mathbb{R}^{n} ; A\right)$ with $\left\{a^{1+i y}: y \in \mathbb{R}\right\}$ relatively weakly compact, then $a=0$.

Proof: Let $\varphi \in \mathcal{F}$, with respect to $A$, and define

$$
(r \otimes \varphi)(f)=\int_{\mathbb{R}^{n}} e^{-i r . s} \varphi(f(s)) d s \in \mathcal{L}(X),
$$

$\left(f \in L^{1}\left(\boldsymbol{R}^{n} ; A\right), r \in \mathbb{R}^{n}\right)$. Then $r \otimes \varphi$ is a (finite-dimensional) irreducible representation of $L^{1}\left(\mathbb{R}^{n} ; A\right)([7$, p.461]), and therefore the spectrum $\sigma(r)$ of 
$(r \otimes \varphi)(a)$ in $\mathcal{L}(X)$ is contained in $\sigma(a)([2$, p.158]), which is an at most countable subset of $\mathbf{R}$, by Theorem 2.2. Now, since $\mathcal{L}(X)$ is finite-dimensional, the spectrum function is continuous on $\mathcal{L}(X)([2, \mathrm{p} .8])$ which implies that the spectral radius $\rho$ is also continuous on $\mathcal{L}(X)$. It follows that $\{\rho(r \otimes \varphi)(a))=$ $\left.\max _{\lambda \in \sigma(r)}|\lambda|: r \in \mathbb{R}^{n}\right\}$ is a connected subset of $\{|\lambda|: \lambda \in \sigma(a)\}$. Moreover, $\lim r_{r \rightarrow \infty}(r \otimes \varphi)(a)=0$, whence we deduce that $\rho((r \otimes \varphi)(a))=0$ for every $r \in \mathbb{R}^{n}$. So, for each $r \in \mathbf{R}^{n}$, the Banach subalgebra of $\mathcal{L}(X)$ generated by the semigroup $\left((r \otimes \varphi)\left(a^{z}\right)\right)_{R e z>0}$ is radical. But this is not possible unless $(r \otimes \varphi)(a)=0$ ([26, Proof of Theorem 5.6, p.80]). Then we have obtained that $\varphi\left(\int_{\mathbb{R}^{n}} e^{-i r \cdot s} a(s) d s\right)=0$ for all $r \in \mathbf{R}^{n}$ and $\varphi \in \mathcal{F}$. Since $A$ is $\mathcal{F}$-semisimple we obtain that $\int_{\mathbb{R}^{n}} e^{-i r . s} a(s) d s=0$ for every $r \in \mathbf{R}$, whence, as usually, by composition with continuous linear functionals on $A$, we deduce that $a=0$.

The above proposition applies to $A=L^{1}(G)$, if $G$ is a (so-called) maximally almost periodic group (see $[19, \mathrm{p} .428]$ ), and this fact gives rise to the following result.

Theorem 3.4. Let $G$ be a central group such that $L^{1}(G)$ has a non-zero analytic semigroup $\left(a^{z}\right)_{R e z>0}$ with $\left\{a^{1+i y}: y \in \mathbb{R}\right\}$ relatively weakly compact in $L^{1}(G)$. Then $G$ contains a compact, open, normal, subgroup $K$ such that $G / K$ is abelian.

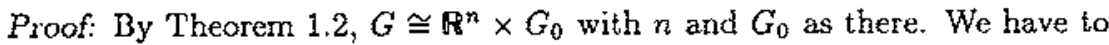
prove that $n=0$. Suppose, if possible, that $n \geq 1$. Since $G_{0}$ is (isomorphic to) a closed subgroup of $G, L^{1}\left(G_{0}\right)$ is semisimple for the set of its finite-dinensional irreducible representations ([21, p.15], [19]). Also, because of $L^{1}(G)=L^{1}\left(\boldsymbol{B}^{n} \times\right.$ $\left.G_{0}\right)=L^{1}\left(\mathbb{R}^{n}\right) \otimes_{\pi} L^{1}\left(G_{0}\right)=L^{1}\left(\mathbb{R}^{n} ; L^{1}\left(G_{0}\right)([2\right.$, p.132]), it is enough to take $A=L^{1}\left(G_{0}\right)$ in Theorem 3.3 to obtain that $a=0$, which is a contradiction.

We write now the converse result announced after Proposition 3.1 .

Corollary 3.5. (i) Let $G$ be a central groutp. Then $G$ contains a compact, open, subgroup if and only if $L^{1}(G)$ has a non-zero analytic semigroup $\left(a^{z}\right)_{k e z>0}$ such that $\left\{a^{\ddagger+i y}: y \in \mathbb{R}\right\}$ is norm compact (or relatively weakly compact) in $L^{1}(G)$.

(ii) Let $G$ be a central and connected group. Then $G$ is compact if and only if $L^{1}\left(G^{\prime}\right)$ has a non-zero analytic semigroup $\left(\alpha^{z}\right)_{R e} z>0$ such that $\left\{a^{1+i y}: y \in \mathbb{R}\right\}$ is norm compact (or relatively weakly compact) in $L^{1}(G)$.

This cerrellary is a consequence of Proposition 3.1 and Theorem 3.4. For part (ii) we need also Theorem 1.3 .

As we have seen, the study of Question $E$ for central groups depends on the study of the existence in $L^{1}(\mathbf{R})$ of analytic semigroups, bounded on $\{\operatorname{Re} z=1\}$. This leadis us to pose the following questions.

Suppresse that 


$$
\left(a^{z}\right)_{R e z>0} \text { such that } \sup _{y \in \mathbb{R}}\left\|a^{1+i y}\right\|<\infty
$$

Question 1. Is $\varphi\left(a^{2+i y}\right)\left(y \in \mathbf{R}, \varphi \in L^{\infty}(\mathbb{R})\right)$ a weakly almost periodic function on $\mathbb{R}$ ?

Question 2. Does (**) imply that, for some non-zero analytic semigroup $\left(b^{z}\right)_{R e x>0}$ in $L^{1}(\mathbb{R}),\left\{b^{1+i y}: y \in \mathbb{R}\right\}$ is relatively weakly compact?

Also, for any connected, locally compact group $G$, we may ask this other question

Question 3. Suppose that there exists a non-zero element $f$ in $L^{1}(G)$ such that the spectrum $\sigma(f)$ is at most countable. Does it follow that $G$ must be compact?

\section{References}

[1] E. Amerio AND G. Prouse, "Almost-periodic functions and functional equations," Van Nostrand, New York, 1971.

[2] B. Auperit, "Propriétés Spectrales des Algèbres de Banach," Lecture Notes in Math. 735, Springer Verlag, Berlin, 1979.

[3] J.M. BACHAR AND OTHERS (ED.), "Radical Banach Algebras and Automatic Continuity," Proc. Long Beach 1981, Lecture Notes in Math. 975, Springer Verlag, Berlin, 1983.

[4] H. BoHR, "Almost Periodic Functions," Chelsea Pub. Co., New York, 1947.

[5] F.F. Bonsall and J. Duncan, "Complete Normed Algebras," SpringerVerlag, Berlin, 1973.

[6] J.C. CANDEAL AND J.E. GALÉ, On the existence of analytic semigroups bounded on the half-disc in some Banach algebras, Bull. London Math. Soc. 21 (1989), 573-576.

[7] T.K. CARNE, Representation theory for tensor products of Banach algebras, Math. Proc. Camb. Phil. Soc. 90 (1981), 445-463.

[8] J. DixMIER, Opérateurs de rang fini dans les représentations unitaires, Pub. Math. I.H.E.S. 6 (1969), 171-204.

[9] W.E. EBERLEIN, Abstract ergodic theorems and weak almost-periodic functions, Trans. Amer. Math. Soc. 67 (1949), 217-240.

[10] W.E. EBERLEIN, The spectrum of weakly almost-periodic functions, Mich. J. Math. 3 (1958), 137-139.

[11] R.E. EDWARDS, "Functional Anelysis : Theory and applications," Holt, Rinehart and Winston, New York, 1965. 
[12] W. EMERSON AND F.P. GREENLEAF, Asymptotic behavior of products $C^{p}=C+\ldots+C$, Trans. Amer. Math. Soc. 145 (1969), 171-204.

[13] J. ESTERLE, Quasimultipliers, representations of $H^{\infty}$, and the closed ideal problem for commutative Banach algebras, in [3].

[14] J. ESTERLE AND J.E. GALÉ, Regularity of Banach algebras generated by analytic scmigroups satisfying some growth conditions, Proc. Amer. Math. Soc. 92 (1984), 377-380.

[15] I. Glicksberg AND K. DE LEeUw, Applications of almost periodic compactifications, Acta Math. 105 (1961), 63-97.

[16] F.P. Greenleaf, "Invariant Means on Topological Groups," Van Nostrand, New York, 1969.

[17] S. Grosser AND M. MOSKOWITz, On central topological groups, Trans. Amer. Math. Soc. 127 (1967), 317-340.

[18] S. Grosser AND M. Moskowitz, Representation theory of central topological groups, Trans. Amer. Math. Soc. 129 (1967), 361-390.

[19] S. Grosser AND M. MOSkowitz, Harmonic analysis on central topological groups, Trans. Amer. Math. Soc. 156 (1971), 419-454.

[20] E. HEwI'T AND K.A. Ross, "Abstract Harmonic Analysis," I, II, Springer Verlag, Berlin, 1963/70.

[21] H. HeYeR, "Probability Measures on Locally Compact Groups," Springer Verlag, Bertin, 1977.

[22] A. HULANICKI, On positive functionals on a group algebra multiplicative on a subalgebra, Studia Math. 37 (1971), 163-171.

[23] A. Hulanicki, Subaigebra of $L^{1}(G)$ associated with Laplacian on a Lie group, Colloquium Math. 31 (1974), 259-287.

[24] R. LARsen, "Banach Algebras," M. Dekker, New York, 1973.

[25] T. PYTLIK, Analytic semigroups in Banach algebras and a theorem of Hilie, Colloguizm Math. 51 (1987), 287-293.

[26] A.M. Sinclair, "Continuous Semigroups in Banach Algebras," London Math. Soc. Lecture Notes Series 63, Cambridge UP, 1982.

[27] J.E. GALÉ, Banach algebras generated by analytic semigroups having compactness properties on vertical lines, Proc. Centre Math. Anal. Austral. Nat. Univ. 21 (1989), 126-143. 